\title{
A Novel Bio-Inspired Technique for Rapid Real-Time Generator Coherency Identification
}

\author{
Jin Wei, Student Member, IEEE, Deepa Kundur, Senior Member, IEEE, \\ and Karen L. Butler-Purry, Senior Member, IEEE
}

\begin{abstract}
Generator coherency identification is establishing itself as an important task to aid in the resistance of cascading failures within wide-area power systems and as a necessary preprocessing stage in real-time control for transient stability. Inspired by flocking behavior in nature, we propose a novel multiflock-based technique to identify generator coherence rapidly within a short observation window. Our measurementbased approach transforms generator data from the observation space to an information space, whereby the generator frequencies and phases characterize the movement and dynamics of boids within multiple flocks. Analysis of the boids' trajectories enables the discrimination of multiple flocks corresponding to coherent generator clusters. We demonstrate the effectiveness of our technique to identify generator coherency rapidly while exhibiting robustness to environmental noise and cyber attack on the 39-bus New England test system and a modified IEEE 118-Bus test system.
\end{abstract}

Index Terms-Flocking model, generator coherency identification, intelligent monitoring.

\section{INTRODUCTION}

A S WE SHIFT toward an era of "smarter" power systems, we witness increased functionality and scale due to load growth and generation expansion. Physical components, increasingly operated under stress in this newly deregulated market, exhibit protracted interarea oscillations detrimental to the goals of maximizing system stability and optimizing power transfer [1]. Wide-area monitoring, protection and control (WAMPAC) systems are designed to address the problem of interarea oscillation. A necessary first step in this process is to first identify the nature of the oscillatory behavior.

Interarea oscillations are a complex phenomenon observed between interconnected synchronous generators in power systems. These oscillations, associated with groups of generators that swing against each other at frequencies below $1 \mathrm{~Hz}$, are

Manuscript received December 2, 2013; revised April 21, 2014; accepted June 29, 2014. Date of publication August 8, 2014; date of current version December 17,2014. This work was supported in part by the U.S. National Science Foundation under Grant ECCS-1028246, and in part by the Natural Sciences and Engineering Research Council of Canada. Paper no. TSG-00894-2013.

J. Wei is with the Department of Electrical and Computer Engineering, University of Akron, Akron, OH 44325 USA (e-mail: jwei@uakron.edu).

D. Kundur is with the Department of Electrical and Computer Engineering, University of Toronto, Toronto, ON M5S 2E4, Canada (e-mail: dkundur@comm.utoronto.ca).

K. L. Butler-Purry is with the Department of Electrical and Computer Engineering, Texas A\&M University, College Station, TX 77843 USA (e-mail: klbutler@tamu.edu).

Color versions of one or more of the figures in this paper are available online at http://ieeexplore.ieee.org.

Digital Object Identifier 10.1109/TSG.2014.2341213 intrinsically nonlinear and dynamic [2]. Generator coherency is used to characterize the observation that generators naturally form clusters, following a disturbance such that generators angles within the same coherent group oscillate faster with respect to one another than those in distinct groups [3].

Much research has been done to analyze interarea oscillation and to identify generator coherency arising from this phenomenon [2]-[10]. Classical techniques can be categorized into three types. The first class identifies generator coherency by analyzing the empirical results of offline simulation [3], [4]; such approaches exhibits high accuracy but are inappropriate for addressing disturbances dynamically in real-time. The second type employs linearized power system models about the power flow equilibrium to reduce complexity [5], but is often unsuitable to represent large or rapid deviations from the equilibrium. The third class introduces the notion of slow coherency arising from interarea oscillations [6]. These methods use singular perturbation to assess time-scale separation of the interarea and local modes, and implement eigenvectorbased methods to identify coherent generator groups. One disadvantage is the inefficiency of these methods when the interarea oscillation is not sufficiently reduced. Furthermore, all three classes require detailed power system topology data, which may not always be available when needed especially for emerging wide area smart grid systems.

To address this problem, measurement-based coherency identification employing phasor measurement units (PMUs) has been proposed. PMUs provides real-time synchronized phasor information of quantities including voltage and current at generator and load busses. PMUs have been developed to measure generator rotor frequencies and phase angles to an accuracy of $0.2 \%$ [11]-[13]. The main advantage of direct PMU monitoring of generators is measurement accuracy resulting in enhanced transmission network reliability as recently surmised [14]-[18]. Moreover, the markets and reliability committee of PJM interconnection [a regional transmission organization (RTO) in the USA] has approved a service agreement of late requiring the installation of PMUs at new interconnections [14]. Thus, it is expected that PMU placement will include generator buses of interest in coherency identification applications.

Under this assumption, Jonsson et al. [7] propose a shorttime Fourier transform (STFT)-based coherency identification method applied to generator frequency measurements. Here, the Fourier coefficient with largest magnitude is used to identify the dominant interarea mode. A sufficiently long observation window is critical for performance hence making 


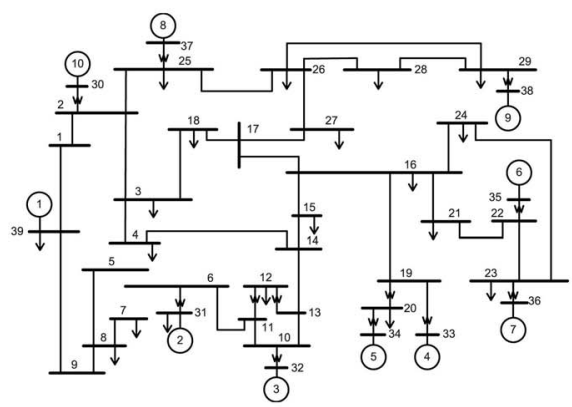

(a)

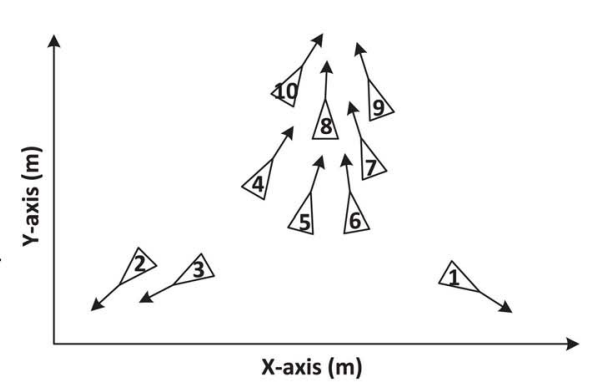

(b)

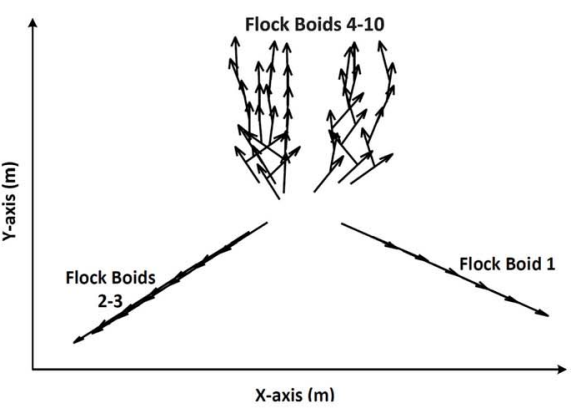

(c)

Fig. 1. (a) New England 39-bus power system. (b) Flocking-based analogy where boids travel through information space. (c) Traveling boids' directed trajectory in the information space.

it inappropriate when only a short duration of data exists upon which to make decisions. The methods proposed in [9], [10] somewhat relax the window length requirement. In [9], the authors employ Koopman modes (KM) derived through spectral analysis of the Koopman operator, a linear operator defined to analyze nonlinear dynamical systems. KM analysis is applied on generator frequency data to characterize nonlinear oscillatory modes for coherency identification. The author in [10] applies the Hilbert-Huang transform on generator phase angle data to identify coherency.

Our research focuses on developing a real-time generator coherency identification technique for the application of transient stability maintenance in the face of serious disturbance. We aim to further shorten the observation window required for generator coherency identification to enable rapid cyberphysical response important in emerging smart grid systems. Initial work by the authors is presented in [19] in which we introduce the notion of applying flocking models to generation coherency identification. In this paper, we extend our work significantly by exploring multiflock models and strategies to enhance robustness in the presence of environmental noise and cyber attack. Built upon the work in [19], this paper achieves three significant contributions.

1) Achieving situational awareness by developing the dynamic criteria to determine the interaction, such as attraction and repulsion, between the boids in the information space.

2) Strengthening the robustness to environmental noise and cyber attack by considering the historical information on the feature similarity between the boids.

3) Comparing the performance of our proposed technique with three other existing techniques in different environmental situations.

Our strategy involves shifting the generator state data from an observation space to an information space that enables better predictability of coherent cluster formation thus reducing the necessary observation window for accurate identification. Moreover, to the best of our knowledge our proposed technique is the first to meet the simultaneous objectives of accuracy, real-time performance, short window length and robustness to corruption. We demonstrate how our technique lends itself to visual display of the results making the approach also suitable for interpretation by a human operator.
In the next section we introduce the coherency identification problem in the context of flocking. In Section III, we discuss our proposed technique. Simulation results are presented in Section IV followed by conclusions in Section V.

\section{Problem Setting}

\section{A. Flocking}

Flocking is a behavior exhibited by groups of birds participating in a shared objective such as flying to a mutual destination. Such behavior enables a collective to accomplish an objective difficult to achieve individually through a combination of cooperation, consensus and informed-adaptation.

The behavior within a single flock has been described by a set of heuristic agent-interaction rules [20], [21].

1) Flock Centering: Boids (bird-like objects) attempt to stay close to nearby flockmates.

2) Velocity Matching: Boids attempt to match velocity with nearby flockmates.

3) Collision Avoidance: Boids avoid collisions with nearby flockmates.

4) Obstacle Avoidance: Boids avoid obstacles often steering away from their goals.

In this paper we aim to detect generator coherency by proposing an analogy between the coherency of generators and the interactions among boids in multiple flocks. We extend a single flocking paradigm to include the characterization of multiple species flocking via a feature similarity rule. Here, a flock boid aims to stay close to those boids representing similar coherency indices and stays apart from boids with dissimilar features much like the flock separation observed for different species in nature.

We assert that a flocking paradigm exhibits the following advantages. The robustness of the flocking process to perturbation and sudden environmental change would make a clustering algorithm based on this analogy more robust to measurement noise and data corruption. Furthermore, the representation of generator data as directed trajectories of boid dynamics enables opportune assessment of future movement and immanent clustering. We emphasize that this predictive quality reduces the need for advanced computation and dramatically decreases the observation time window in contrast to existing techniques. 


\section{B. Multiflock-Based Coherency Identification}

Our coherency identification technique makes use of a transformation from the observation space to an information space where decisions on generator coherence are directly made. The bulk of our proposed technique involves the mapping from these domains and is detailed in Section III.

As illustrated in Fig. 1(a)-(c), for the New England 39-bus power system, each boid in the flocking scenario embodies the state information of a corresponding generator of the power system. For $C$ generators indexed as $i=1,2, \ldots, C$, Boid $i$ embodies the information of Generator $i$. In nature, boids of the same species are within the same flock. In our technique, the species feature of each Boid $i$ is determined by the information describing Generator $i$ 's status in the observation space and is defined as

$$
\left\{\begin{array}{l}
\mathcal{I}_{i}^{1}(k)=\theta_{i}(k) \\
\mathcal{I}_{i}^{2}(k)=\omega_{i}(k) \\
\mathcal{I}_{i}^{3}(k)=\delta_{i}(k)
\end{array}\right.
$$

where $\mathcal{I}_{i}(k)=\left[\mathcal{I}_{i}^{1}(k) \mathcal{I}_{i}^{2}(k) \mathcal{I}_{i}^{3}(k)\right]^{T}, \theta_{i}(k)$ and $\omega_{i}(k)$ are the phase angle and the normalized frequency, respectively, of the $i$ th generator at the time step $t=k$ that are obtained directly from PMU information, and $\delta_{i}(k)$ is the acceleration of the $i$ th generator at the time step $t=k$ estimated from the current and historical values of $\omega_{i}(k)$ as we later discuss.

In the information space, we describe the dynamic state of each boid as $\mathcal{S}_{i}(k)=\left[\mathbf{p}_{i}(k), \mathbf{v}_{i}(k)\right]^{T}$, where $\mathbf{p}_{i}(k)$ denotes Boid $i$ 's position initialized randomly, and $\mathbf{v}_{i}(k)$ is Boid $i$ 's velocity initialized to $\mathbf{0}$. We also consider two boids to be neighbors at time step $t=k$ if the distance between them is less than the predetermined threshold $d_{c}$, and thus define the index set of neighbors for the $i$ th boid as follows: $\mathcal{N}_{i}(k)=$ $\left\{\forall j \neq i \mid\left\|\mathbf{p}_{i}(k)-\mathbf{p}_{j}(k)\right\|<d_{c}\right\}$, which is comprised of the indices of all of Boid $i$ 's neighbors.

The boid positions are initialized to random locations. Then, each new generator data set in the observation space affects the movement of its associated boid in the observation space as shown in Fig. 1(c). Despite its initial values, we assert based on empirical experience that, over time, the boids' relative positions and velocities converge to invariant clusters through the interaction with neighboring boids. The boids assemble into multiple flocks, largely determined by their species feature that must be appropriately defined such that groups within the configuration correspond to generator clusters. For example, by observing Fig. 1(c), we can conclude that Boids 2 and 3 are in the same flock and Boids 4 to 10 constitute another flock, which indicates a generator coherency following a serious fault of $\left\{G_{1}\right\},\left\{G_{2}, G_{3}\right\}$, and $\left\{G_{4}, G_{5}, \ldots, G_{10}\right\}$.

The authors would like to clarify that flocking model is not employed to directly describe the generator dynamics. The mapping from the observation space to the information space is nonlinear such that the boids behavior is influenced by the generator state, which is used to compute appropriate attraction/repulsion to other boids hence instigating movement. Thus, a boid does not directly represent a generator, but may be considered to have characteristics that carry the state information. Our model enables a visual identification of generator grouping as the formation of distinct flocks within a multiflock system, but this movement does not directly reflect the raw generator dynamics.

\section{Multiflock-Based Generator COHERENCY IDENTIFICATION}

We assume that each generator has a phasor measurement unit (PMU) that acquires its phase and frequency information and communicates with a control center [7], [22]. To identify generator coherency using our approach, the control center would map the generator frequency and phase to boid dynamics in the information space and then analyze the interactions between these boids. The corresponding generator frequency and phase information of a boid relative to that of another neighboring boid represents a feature, which in turn determines the nature of its interaction with its neighbor. Let $\chi_{i j}^{k}=\mathbf{p}_{j}(k)-\mathbf{p}_{i}(k)$ be the Euclidean displacement between the Boid $i$ and $j$ in the information space at time $t=k$. We consider the boids' interaction at each time step $t=k$ to be characterized in terms of a weighted combination of intraflock interaction and interflock interaction modeled through the use of a potential function $V^{k}\left(\chi_{i j}^{k}\right)$, which is a function of variable $\chi_{i j}^{k}$ and defined as

$$
V^{k}\left(\chi_{i j}^{k}\right)=\sum_{l=1}^{3} w_{l} V_{l}^{k}\left(\chi_{i j}^{k}\right)
$$

where $V_{1}^{k}$ models the intraflock centering and collision avoidance, $V_{2}^{k}$ represents the intraflock velocity matching, and $V_{3}^{k}$ is used to account for interflock obstacle avoidance. Thus, the relative similarity in state of a pair of generators is employed to produce a potential between the corresponding boids via $V^{k}$, which imposes accelerations on the boids to determine the boids' pairwise interactions and trajectories. The acceleration caused by a force Boid $j$ imposes on Boid $i$ is computed by differentiating the potential function $V^{k}$ with respect to the relative position vector between Boid $i$ and $j$. Thus, the overall acceleration of Boid $i$ would be a vector sum of the individual accelerations caused by each of Boid $i$ 's neighbors.

\section{A. Multiflock Modeling}

1) Feature Similarity: The potential functions can be computed for a pair of neighboring boids only after the feature similarity is calculated. We compute a species feature similarity between neighboring boids as follows. For $j \in \mathcal{N}_{i}(k)$, the indices of all Boid $i$ neighbors

$$
\zeta_{i j}(k)=\left|\sum_{n=1}^{3} \alpha_{n}\left(\mathcal{I}_{i}^{n}(k)-\mathcal{I}_{j}^{n}(k)\right)\right|
$$

where $\left\{\alpha_{n}\right\}$ is a positive scalar weight influencing the impact of information class $n$ on boid interaction. As evident, $\zeta_{i j}(k)$ is smaller for higher feature similarity.

Based on (3), we calculate a dynamic threshold $\zeta_{t h}(k)$ and determine whether a pair of neighboring boids are part of the same flock by comparing their feature similarity with this threshold value; the procedure is detailed in Table I. First, we define set $\zeta(k)$, which is a collection of the feature similarities between all the pairwise neighboring boids at time 
TABLE I

NEIGHBORING FLOCKMATE RELATIONSHIPS

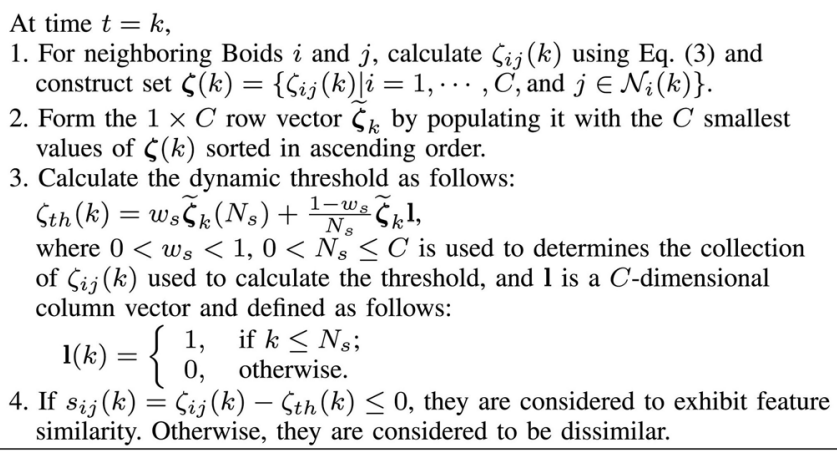

$t=k$, and then obtain a sorted row vector $\widetilde{\zeta}_{k}$ consisting of the $C$ smallest elements of $\zeta(k)$ in ascending order. Second, we select the first $N_{s}$ smallest feature similarity values (i.e., the first $N_{s}$ elements of $\widetilde{\zeta}_{k}$ ) to compute the dynamic threshold $\zeta_{t h}(k)$ via a weighted summation of the maximum and the mean of the smallest $N_{s}$ feature similarity values. Finally, we conclude that Boids $i$ and $j$ have high similarity if the sign of $s_{i j}(k)=\zeta_{i j}(k)-\zeta_{t h}(k) \leq 0$. The reader should note that the value of $s_{i j}(k)$ will be employed to compute each potential function for a pair of neighbors.

2) Intraflock Centering and Collision Avoidance: Intraflock centering and collision avoidance involves the attraction of boids to one another such that they are in the same geographic proximity but are noncolliding. We model this for boids within the same flock through interboid forces that depend on relative displacement and informational similarity. Thus, for $s_{i j}(k) \leq 0$, Boids $i$ and $j$ are assumed to exhibit cohesion; they are attracted to one another up to a desired position distance $d^{*}$ (at which point we consider the attraction strength to decrease to zero) and then if any closer, slightly repel each other to avoid collision. The higher informational similarity between the boids, the stronger (weaker) the strength (repulsion) when their distance is above (below) $d^{*}$.

We design a nonnegative potential energy function $V_{1}^{k}\left(\chi_{i j}^{k}\right)$ for all $(i, j)$ such that $j \in \mathcal{N}_{i}(k)$ and $s_{i j}(k) \leq 0$, which reaches its minimum only when its argument $\chi_{i j}^{k}$ implies a position separation between Boids $i$ and $j$ of distance $d^{*}$

$$
\begin{aligned}
V_{1}^{k}\left(\chi_{i j}^{k}\right)= & \sum_{i=1}^{C} \sum_{j \in \mathcal{N}_{i}(k)}\left\{\gamma_{1} / \gamma_{2} \cdot \exp \left[\gamma_{2} s_{i j}(k)\left(\left\|\chi_{i j}^{k}\right\|_{\sigma}-\left\|d^{*}\right\|_{\sigma}\right)\right]\right. \\
& \left.-\gamma_{1} s_{i j}(k)\left(\left\|\chi_{i j}^{k}\right\|_{\sigma}-\left\|d^{*}\right\|_{\sigma}\right)\right\}
\end{aligned}
$$

where $\gamma_{1}$ and $\gamma_{2}$ are positive parameters, and $\|\cdot\|_{\sigma}$ denotes the $\sigma$-norm $\|\mathbf{x}\|_{\sigma}=\frac{1}{\epsilon}(\sqrt{1+\epsilon\|\mathbf{x}\|}-1)$ which is used to guarantee the potential energy function $V_{1}^{k}\left(\chi_{i j}^{k}\right)$ is always differentiable, especially when $\chi_{i j}^{k}=0$. If $s_{i j}(k)>0$ then $V_{1}^{k}\left(\chi_{i j}^{k}\right)=0$.

Using (4), we calculate the corresponding action function $\mathbf{g}_{i, j}^{1}(k)$, which is equivalent to the acceleration caused by the potential energy function $V_{1}^{k}$, by differentiating $V_{1}^{k}$ with respect to relative displacement $\chi_{i j}^{k}[23]$ to give

$$
\begin{aligned}
\mathbf{g}_{i, j}^{1}(k) & =\nabla_{\chi_{i j}^{k}} V_{1}^{k}\left(\chi_{i j}^{k}\right) \\
& \left.=\frac{\gamma_{1} \cdot s_{i j}(k) \cdot\left(\chi_{i j}^{k}\right)}{\sqrt{1+\epsilon\left\|\chi_{i j}^{k}\right\|^{2}}}\left\{\exp \left[\gamma_{2} s_{i j}(k)\left\|\chi_{i j}^{k}\right\|_{\sigma}-\left\|d^{*}\right\|_{\sigma}\right)\right]-1\right\}
\end{aligned}
$$

for all $(i, j)$ such that $j \in \mathcal{N}_{i}(k)$ and $s_{i j}(k) \leq 0$ and $\mathbf{g}_{i, j}^{1}(k)=0$ otherwise.

3) Velocity Matching: In a flock, flockmates typically demonstrate alignment of their velocities. It is well established that this can be modeled as a distributed consensus problem in which the boids represent the vertices of a dynamic graph and the associated edge weights determine the degree of interaction to achieve alignment [24]. Letting $\mathbf{A}$ be the dynamic graph's associated adjacency matrix with elements $a_{i j}(k)$, we design the following potential energy function for all $(i, j)$ such that $j \in \mathcal{N}_{i}(k)$ and $s_{i j}(k) \leq 0$ :

$$
V_{2}^{k}\left(\chi_{i j}^{k}\right)=\left(\chi_{i j}^{k}\right)^{T} \mathbf{L} \chi_{i j}^{k}
$$

where $\mathbf{L}$ is the graph's associated Laplacian matrix: $\mathbf{L}=\Delta(\mathbf{A})-\mathbf{A}$ and $\Delta \mathbf{A}$ is a diagonal matrix with the $i$ th diagonal element as $\sum_{j=1}^{n} a_{i j}(k)$. For $s_{i j}(k)>0, V_{2}^{k}\left(\chi_{i j}^{k}\right)=0$.

In our framework, the interaction that achieves alignment is designed to be constant when the relative displacement $\chi_{i j}^{k}$ is below the threshold $d_{c}$ (i.e., $j \in \mathcal{N}_{i}(k)$ ) and reduces to $\mathbf{0}$ otherwise. Therefore, we assign the adjacency matrix elements of such a graph in our formulation as

$$
a_{i j}(k)=\rho_{h}\left(\left\|\chi_{i j}^{k}\right\|_{\sigma} /\left\|d_{c}\right\|_{\sigma}\right)
$$

for $j \in \mathcal{N}_{i}(k)$ where $h \in[0,1)$ is a parameter, and

$$
\rho_{h}(x)= \begin{cases}1, & x \in[0, h) \\ \frac{1}{2}[1+\cos (\pi(x-h) /(1-h))], & x \in[h, 1] \\ 0, & \text { otherwise. }\end{cases}
$$

Using (6) and (7), we determine the corresponding acceleration to be

$$
\mathbf{g}_{i, j}^{2}(k)=a_{i j}(k) \chi_{i j}^{k}
$$

for all $(i, j)$ such that $j \in \mathcal{N}_{i}(k)$ and $s_{i j}(k) \leq 0$ and $\mathbf{g}_{i, j}^{2}(k)=0$ otherwise, as desired.

4) Obstacle Avoidance: For $s_{i j}(k)=\zeta_{i j}(k)-\zeta_{t h}(k)>0$, Boids $i$ and $j$ are considered to exhibit low feature similarity. Their interaction is modeled as a mutual avoidance in which each considers the other to be an obstacle in their flock's trajectory. We model the corresponding repulsion strength to be dependent on their degree of dissimilarity and their relative distance to one another; the higher the dissimilarity the stronger the repulsion up to a distance threshold $d_{r}$ at which point their repulsion strength decreases to 0 . Thus, we design the following potential energy function for all $(i, j)$ such that 
TABLE II

BOID DYNAMICS

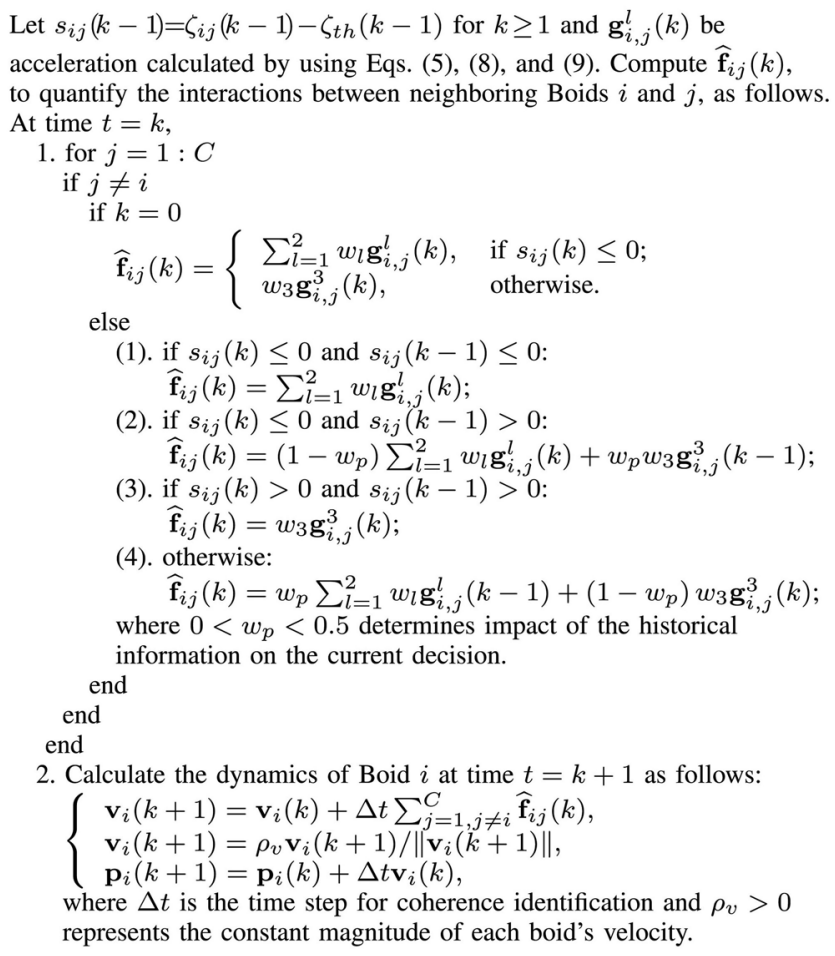

$j \in \mathcal{N}_{i}(k)$ and $s_{i j}(k)>0$ :

$V_{3}^{k}\left(\chi_{i j}^{k}\right)= \begin{cases}\frac{\gamma_{3}}{2} \sum_{i=1}^{C} \sum_{j \in \mathcal{N}_{i}(k)} s_{i j}(k)\left(\left\|\chi_{i j}^{k}\right\|_{\sigma}-\left\|d_{r}\right\|_{\sigma}\right)^{2}, \\ 0, & \text { if }\left\|\chi_{i j}^{k}\right\| \leq d_{r} \\ 0 & \text { otherwise }\end{cases}$

where $\gamma_{3}$ is a positive parameter. $V_{3}^{k}\left(\chi_{i j}^{k}\right)=0$ for $s_{i j}(k) \leq 0$.

We calculate the acceleration caused by $V_{3}^{k}$ as

$$
\begin{aligned}
\mathbf{g}_{i, j}^{3}(k) & =\nabla_{\chi_{i j}^{k}} V_{3}^{k}\left(\chi_{i j}^{k}\right) \\
& =\gamma_{3} s_{i j}(k)\left(\left\|\chi_{i j}^{k}\right\|_{\sigma}-\left\|d_{r}\right\|_{\sigma}\right)
\end{aligned}
$$

for all $(i, j)$ such that $j \in \mathcal{N}_{i}(k)$ and $s_{i j}(k) \leq 0$ and $\mathbf{g}_{i, j}^{3}(k)=0$ otherwise.

\section{B. Coherent Generator Identification}

To ensure that the boids' trajectories are always within the field of view, in our framework the interaction between the boids only affects the direction, rather than magnitude of the boids' velocity. Thus each active boid traveling in the information space has a velocity with constant magnitude and varying direction. Furthermore, the continuity of the generators' measurements in the observation space and the boids' movement in the information space indicate a continuous nature of the interaction amongst the traveling boids. Therefore, the interaction at current time step $t=k$ can be predicted by the value at the previous time step $t=k-1$ with certain prediction accuracy $w_{p}$. Based on this fact, we improve the robustness of
TABLE III

Main Variables Used in Proposed Approach

\begin{tabular}{c|c|c|c}
\hline $\mathcal{I}_{i}$ & $\theta_{i}$ & $\omega_{i}$ & $\delta_{i}$ \\
\hline $\begin{array}{c}\text { Boid } i \text { 's carried } \\
\text { information }\end{array}$ & $\begin{array}{c}\text { Generator } i \text { 's } \\
\text { phase angle }\end{array}$ & $\begin{array}{c}\text { Generator } i \text { 's } \\
\text { frequency }\end{array}$ & $\begin{array}{c}\text { Generator } i \text { 's } \\
\text { acceleration }\end{array}$ \\
\hline \hline $\mathcal{S}_{i}$ & $\mathbf{p}_{i}$ & $\mathbf{v}_{i}$ & $\zeta_{i j}$ \\
\hline $\begin{array}{c}\text { State of } \\
\text { Boid } i\end{array}$ & $\begin{array}{c}\text { Position of } \\
\text { Boid } i\end{array}$ & $\begin{array}{c}\text { Velocity of } \\
\text { Boid } i\end{array}$ & $\begin{array}{c}\text { Feature similarity } \\
\text { between Boids } \\
i \text { and } j\end{array}$ \\
\hline \hline $\begin{array}{c}\text { Relative feature } \\
\text { similarity with } \\
\text { respective to } \\
\text { threshold } \chi_{t h}\end{array}$ & $\begin{array}{c}\text { Index collection } \\
\text { of Boid } i \text { 's } \\
\text { neighbors }\end{array}$ & $\begin{array}{c}\text { Distance } \\
\text { between Boids } \\
i \text { and } j\end{array}$ & $\begin{array}{c}\text { Acceleration of } \\
\text { Boid } i \text { caused } \\
\text { by interaction } \\
\text { with Boid } j \\
\text { based on } V_{l}\end{array}$ \\
\hline
\end{tabular}

TABLE IV

Parameters USEd in Proposed Approach

\begin{tabular}{l|l|l||l|l|l}
\hline Parameters used & $\gamma_{1}$ & 1 & Positive weights & $\alpha_{1}$ & 8 \\
in potential & $\gamma_{2}$ & 0.5 & for boids' carried & $\alpha_{2}$ & 1 \\
functions & $\gamma_{3}$ & $1 / 6$ & information & $\alpha_{3}$ & 3 \\
\hline Positive weights & $w_{1}$ & 350 & Distance threshold for $V_{2}$ & $d_{c}$ & 10 \\
\cline { 4 - 6 } $\begin{array}{l}\text { for potential } \\
\text { functions }\end{array}$ & $w_{2}$ & 100 & Distance threshold for $V_{3}$ & $d_{r}$ & 8 \\
\cline { 4 - 6 } & $w_{3}$ & 750 & Desired distance & $d^{*}$ & 0.5 \\
\hline $\begin{array}{l}\text { Number of boids } \\
\text { used to get } \boldsymbol{\chi}_{t h}\end{array}$ & $N_{s}$ & 10 & $\begin{array}{l}\text { Parameter used } \\
\text { to get } \boldsymbol{\chi}_{t h}\end{array}$ & $w_{s}$ & 0.6 \\
\hline $\begin{array}{l}\text { Impact of history } \\
\text { information }\end{array}$ & $w_{p}$ & 0.2 & $\begin{array}{l}\text { Normalized magnitude } \\
\text { of velocity }\end{array}$ & $\rho_{v}$ & $10^{3}$ \\
\hline
\end{tabular}

our technique to potential measurement noise and cyber attack by accounting for the consistency in the nature of interaction at the current time step $t=k$ with the previous time instant $t=k-1$. Specifically, if the signs of $s_{i j}(k-1)$ and $s_{i j}(k)$ are consistent, the boid's acceleration is calculated based on the interaction achieved at $t=k$, otherwise, the boid's acceleration is calculated based on two components: the first component is related to the interaction achieved at $t=k$ (based on the observation data at this current time step) and the second component is the predicted interaction based on the trajectory set at $t=k-1$. These components are weighted via the prediction accuracy parameter $w_{p}$. The resulting model of Boid $i$ dynamics is detailed in Table II.

The state of each boid $\mathcal{S}_{i}(k)=\left[\mathbf{p}_{i}(k), \mathbf{v}_{i}(k)\right]^{T}$ is used to plot its trajectory in time during the observation period. From this graph, we can easily obtain the grouped arrangement of the boids indicating coherent clusters of generators. To better elucidate our technique, we summarize variables in Table III and list parameters in Table IV. In the next section we demonstrate how our technique effectively determines coherent generator clusters under a variety of faults.

\section{Simulations and Performance Assessment}

We evaluate the effectiveness of our generator coherency identification technique by using the New England 39-Bus test system as shown in Fig. 1(a) and detailed in [25] consisting of $C=10$ generators, which we simulate with MATLAB/Simulink. The average computation time of our proposed method is $0.11 \mathrm{~s}$ using an AMD FX(tm)-8320 Eight-Core Processor and 16 GB RAM. This can be dramatically reduced with dedicated and more advanced processors. The New England 39-Bus test system is widely used for 


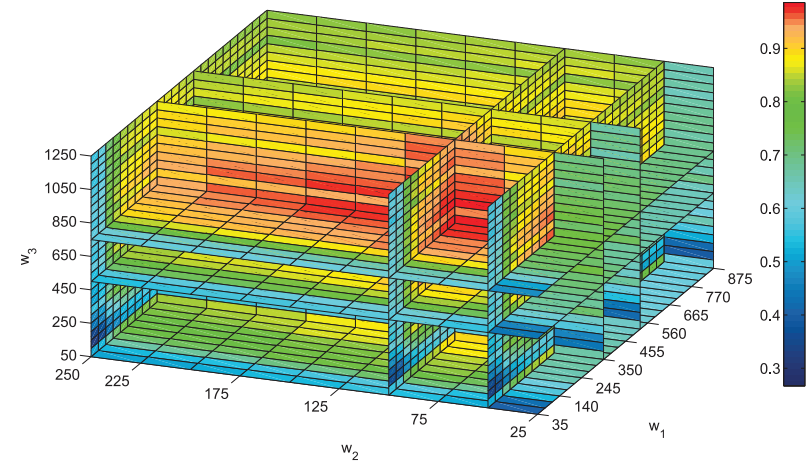

Fig. 2. Probabilities of successfully identifying the generator coherency in one trial by selecting different joint parameters $\left\{w_{1}, w_{2}, w_{3}\right\}$.

studying the complex situations in multiarea power system [26], [27], such as generator coherency presented by inter and intraarea oscillations [9], [28], [29]. To demonstrate the performance of our technique on more complicated generator coherency scenario, in the last case study we implement the simulations on a modified IEEE 118-bus test system, which consists of $C=20$ generators.

For the New England 39-Bus test system, we consider three situations representing an ideal environment, one with white noise, and one with false data injection. In each situation, we employ PMUs with a sampling frequency of $50 \mathrm{~Hz}$ and set the observation window length to $T=100 \mathrm{~ms}$, which is selected empirically through trial and error for different test faults, and $T=500 \mathrm{~ms}$, which is used to evaluate the performances of different techniques. For the modified IEEE 118-bus test system, the situation representing an ideal environment is considered. In the simulation, we employ PMUs with a sampling frequency of $50 \mathrm{~Hz}$ and set the observation window length to $T=100 \mathrm{~ms}$, which starts immediately after the fault in the system is cleared. The reader should note that it was found through tests on the IEEE 9-bus, IEEE 14-bus, New England 39-Bus and modified IEEE 118-bus test systems that the observation window length depends on the test system's complexity and PMU sampling rate. Specifically, higher complexity systems and PMU sampling rates below $50 \mathrm{~Hz}$ generally require longer observation windows.

We conclude the generator coherency determination based on the dominant conclusion of the boid groupings amongst the ten trials. The essential idea of our proposed technique is to identify the generator coherency within a very short observation window by analyzing the boids' trajectories in the corresponding information space. Since the boids' trajectories are achieved through their interaction which is partially dependent on their pairwise distances, the initial positions of the boids may affect their trajectories within a short time duration and hence impact on the rapid generator coherency identification. In order to reduce this uncertainty, in each case study, we run 10 independent trials in each of which the positions of the boids are initialized randomly in the information space.

To assess the performance of our approach, we compare our technique with the classical modal analysis-based scheme by Chow [6] as well as two measurement-based methods

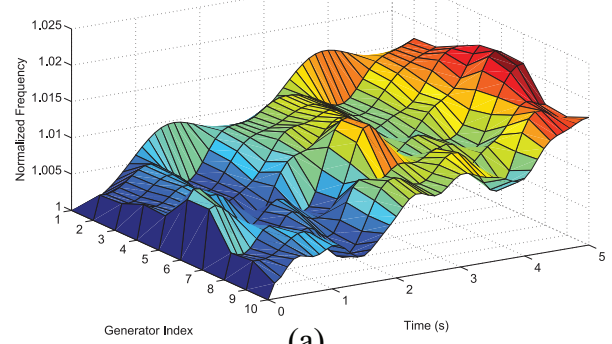

(a)

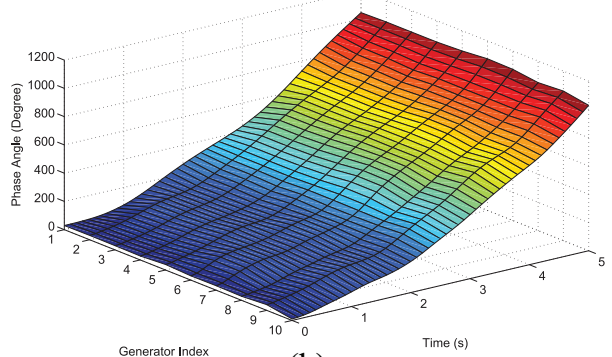

(b)

Fig. 3. (a) Normalized rotor frequencies. (b) Phase angles versus time of $10 \mathrm{~s}$.

proposed in [7] and [9]. The parameters, selected empirically under ideal conditions, are listed in Table IV. We employ the trial-and-error method and implement the judicious empirical analysis on the performance of our proposed technique by selecting different parameter values. For example, the selection of joint parameters $\left\{w_{1}, w_{2}, w_{3}\right\}$ representing the positive potential weights is illustrated in Fig. 2. The $x$-, $y$-, and $z$ axis of Fig. 2 represent the possible values for $\left\{w_{1}, w_{2}, w_{3}\right\}$, respectively, and the color data indicates the probability of successfully identifying the generator coherency in one trial when one of the parameters is fixed at a certain value. We observe that there are multiple parameter assignments to achieve that a probability of success above $95 \%$ displayed in the red and orange colors. Selection $\left\{w_{1}, w_{2}, w_{3}\right\}=\{350,100,750\}$ is used for the simulations in our work.

\section{A. Ideal Environment}

In this section, we consider the situation in which the PMU measurements used for the generator coherency determination have high accuracy.

1) Case 1: We assume a three-phase short circuit fault to occur immediately outside Bus 21 on Line $21-22$ at $t=0$. The fault is cleared by opening the associated Line $21-22$ at $t=100 \mathrm{~ms}$. The generators' normalized rotor frequencies and phase angles over a period of $5 \mathrm{~s}$ are shown in Fig. 3. We observe that the generator coherency involving the following groups: $\left\{G_{1}\right\},\left\{G_{6}, G_{7}\right\}$, and $\left\{G_{2}, G_{3}, G_{4}, G_{5}, G_{8}, G_{9}, G_{10}\right\}$.

Since the transient stability maintenance is time-critical, there is only a brief observation period to determine the generator coherency. Fig. 4 shows the boid trajectories for the observation time window length $T=100 \mathrm{~ms}$, which starts immediately after the fault is cleared, achieved in one trial which achieves the dominant result on boid grouping. The arrows on each trajectory indicates the velocity direction of each boid. From the trajectories shown in Fig. 4, we observe 


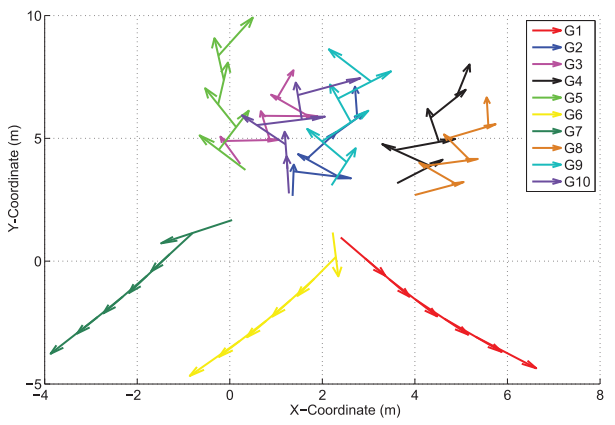

(a)

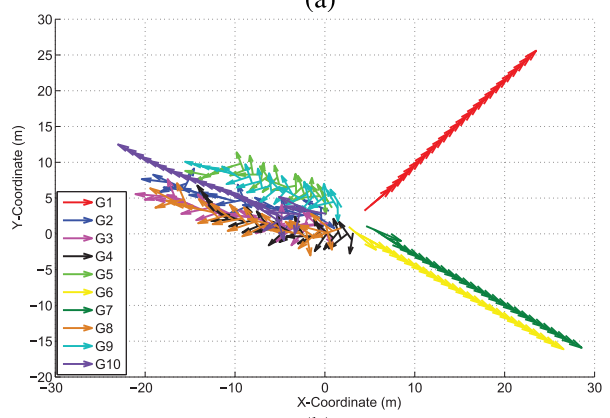

(b)

Fig. 4. Trajectories of the boids with the observation time windows of (a) $100 \mathrm{~ms}$ and (b) $500 \mathrm{~ms}$.

that the boids associated with $G_{6}$ and $G_{7}$ travel together as well as those associated with $\left\{G_{2}, G_{3}, G_{4}, G_{5}, G_{8}, G_{9}, G_{10}\right\}$. Thus, we visually conclude that the boids constitute three flocks identifying coherent generator groups.

For consistent comparison, the modal analysis method of [6], the STFT method of [7] and the KM-approach of [9] are first implemented with the same observation time window length of $100 \mathrm{~ms}$ and PMU sampling of $50 \mathrm{~Hz}$. The modal analysis method uses singular perturbation analysis to achieve an interarea oscillation of $0.43 \mathrm{~Hz}$ and uses an eigenvetor-based algorithm to obtain the slow eigensubspace matrix

$$
V_{s}=\left[\begin{array}{ccccc}
0.32 & 0.32 & 0.32 & 0.32 & 0.32 \\
-0.30 & 0.23 & 0.24 & 0.35 & 0.40 \\
0.32 & 0.32 & 0.32 & 0.32 & 0.32 \\
0.41 & 0.40 & 0.23 & 0.31 & 0.20
\end{array}\right]
$$

based on which the coherent groups of generators are incorrectly identified as $\left\{G_{1}\right\}$, and $\left\{G_{2}, \ldots, G_{10}\right\}$. However, the reader should note that with a longer observation window of $500 \mathrm{~ms}$, this method computes interarea oscillations $0.27 \mathrm{~Hz}$ and $0.67 \mathrm{~Hz}$ and obtains a slow eigensubspace matrix from which coherent groups of generators are correctly identified as $\left\{G_{1}\right\},\left\{G_{6}, G_{7}\right\}$, and $\left\{G_{2}, G_{3}, G_{4}, G_{5}, G_{8}, G_{9}, G_{10}\right\}$. The above simulations show that the modal analysis method achieves accurate identification of generator coherency when the observation time window is long enough such that interarea oscillations are sufficiently damped. The performance of this method degrades when the observation window is short.

The results of [7] where the relative phases of the largest magnitude nonzero Fourier coefficients of each interarea mode are computed are shown in Table V. Since the observation window length used here is much less than the window
TABLE V

Phase Angle Comparison Results (Degree)

\begin{tabular}{c|c|c|c|c|c}
\hline Time Window & G1 & G2 & G3 & G4 & G5 \\
\hline $100 \mathrm{~ms}$ & 0 & -95.61 & -67.37 & -53.18 & -77.75 \\
$500 \mathrm{~ms}$ & 0 & -5.86 & -8.91 & -27.87 & -5.80 \\
\hline \hline Time Window & G6 & G7 & G8 & G9 & G10 \\
\hline $100 \mathrm{~ms}$ & 20.88 & 37.67 & -51.15 & -64.28 & -1.51 \\
$500 \mathrm{~ms}$ & 35.38 & 20.66 & -19.22 & -12.52 & -26.01 \\
\hline
\end{tabular}

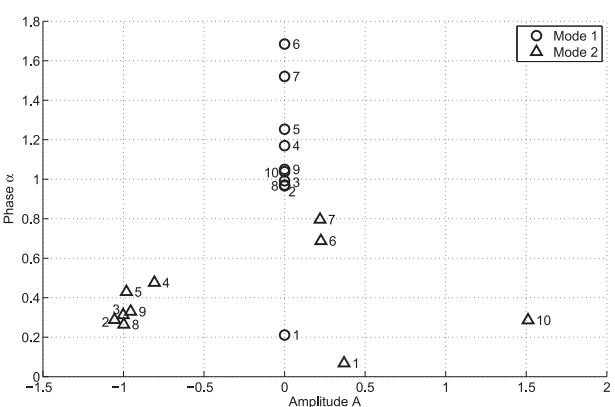

(a)

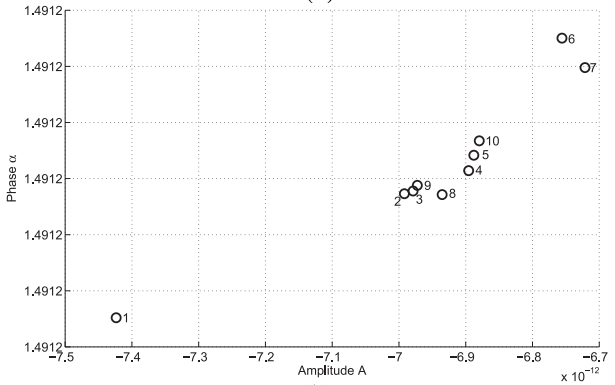

(b)

Fig. 5. Distribution of amplitude coefficients $A_{i j}$ and initial phases $\alpha_{i j}$ for the dominant KMs with the observation time windows of (a) $100 \mathrm{~ms}$ and (b) $500 \mathrm{~ms}$.

length, $100 \mathrm{~s}$, selected in [7], it is reasonable to obtain the phase angle comparison $\Delta \boldsymbol{\varphi}_{k, d o m}$ at one time index. The phase comparison results in Table $\mathrm{V}$ incorrectly indicates that the generator coherency is $\left\{G_{1}, G_{10}\right\}\left\{G_{6}, G_{7}\right\}$, and $\left\{G_{2}, G_{3}, G_{4}, G_{5}, G_{8}, G_{9}\right\}$ with the time window of $100 \mathrm{~ms}$, and correctly as $\left\{G_{1}\right\},\left\{G_{6}, G_{7}\right\}$, and $\left\{G_{2}, G_{3}, G_{4}, G_{5}, G_{8}, G_{9}, G_{10}\right\}$ with time window $500 \mathrm{~ms}$. Thus, the method of [7] exhibits limited performance for short observation windows.

The KM-based method of [9] is implemented to achieve the domain KM by analyzing the generators' frequency measurement. When the time window is $100 \mathrm{~ms}$, there are two dominant KMs and Fig. 5(a) plots the initial phase $\alpha_{i j}$ versus amplitude coefficients $A_{i j}$ of $\mathrm{KM} j$ for $G_{i}$. Based on the distribution of $A_{i j}$ and $\alpha_{i j}$ shown in Fig. 5(a), we can identify the generator coherency as $\left\{G_{1}\right\}\left\{G_{6}, G_{7}\right\}$, and $\left\{G_{2}, G_{3}, G_{4}, G_{5}, G_{8}, G_{9}\right\}$, and the grouping for $G_{10}$ is ambiguous. When the time window is $500 \mathrm{~ms}$, there is one dominant $\mathrm{KM}$ and the distribution of $A_{i j}$ and $\alpha_{i j}$ is shown Fig. 5(b), from which we can correctly conclude the coherent groups of generators as $\left\{G_{1}\right\}\left\{G_{6}, G_{7}\right\}$, and $\left\{G_{2}, G_{3}, G_{4}, G_{5}, G_{8}, G_{9}, G_{10}\right\}$. Thus, the method of [9] also exhibits limited performance for short observation windows.

2) Case 2: We consider that a three-phase fault occurs in the middle of the Line $14-15$ at time $t=0 \mathrm{~s}$ and the fault is cleared at $t=100 \mathrm{~ms}$, post-Critical Clearing 


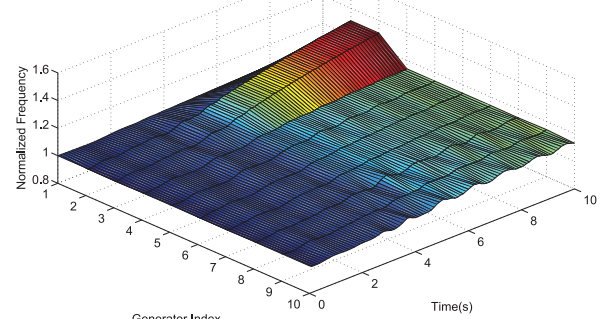

(a)

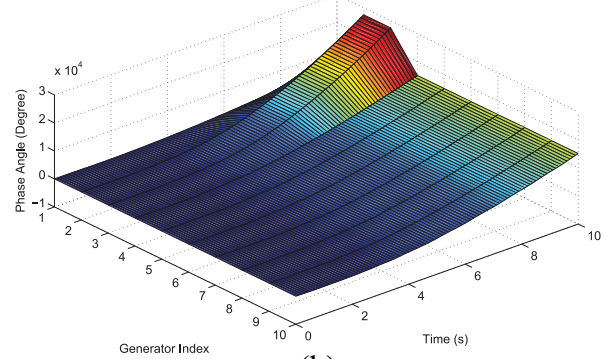

(b)

Fig. 6. (a) Normalized rotor frequencies. (b) Phase angles versus time of $10 \mathrm{~s}$.

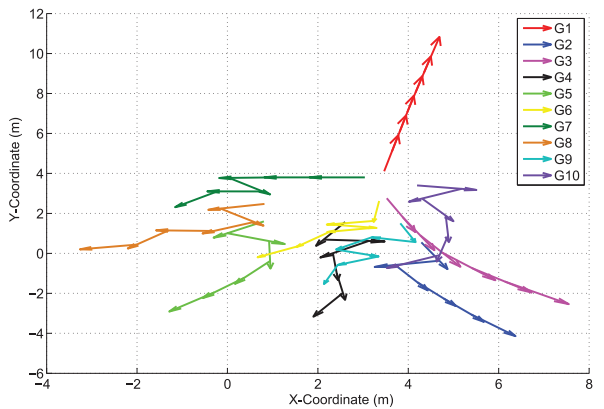

(a)

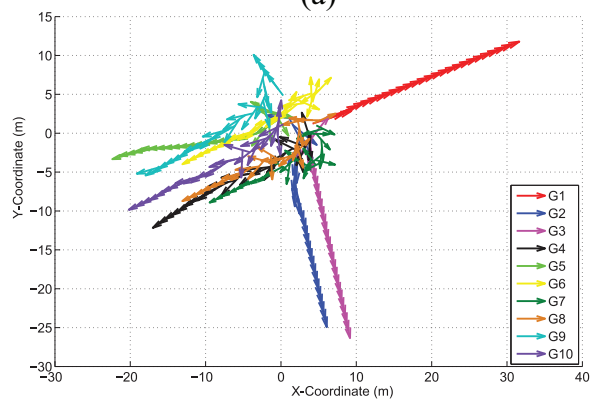

(b)

Fig. 7. Trajectories of the boids with the observation time windows of (a) $100 \mathrm{~ms}$ and (b) $500 \mathrm{~ms}$.

Time (CCT). The generators' normalized rotor frequencies and phase angles over a period of $10 \mathrm{~s}$ are shown in Fig. 6 revealing the following true coherent clusters groups: $\left\{G_{1}\right\},\left\{G_{2}, G_{3}\right\}$, and $\left\{G_{4}, G_{5}, \ldots, G_{10}\right\}$. The boid trajectories of one trial of our proposed technique achieve the dominant result shown in Fig. 7. As evident, the boid associated with $G_{1}$ travels away from the others that form two groups, one corresponding to $G_{2}$ and $G_{3}$, and the other to $G_{4}$ to $G_{10}$.

With the observation time window of $100 \mathrm{~ms}$, the modal analysis method [6] achieves interarea oscillation $0.42 \mathrm{~Hz}$ and mistakenly identifies the coherent groups of generators as $\left\{G_{1}\right\}$, and $\left\{G_{2}, \ldots, G_{10}\right\}$. With an observation time window of

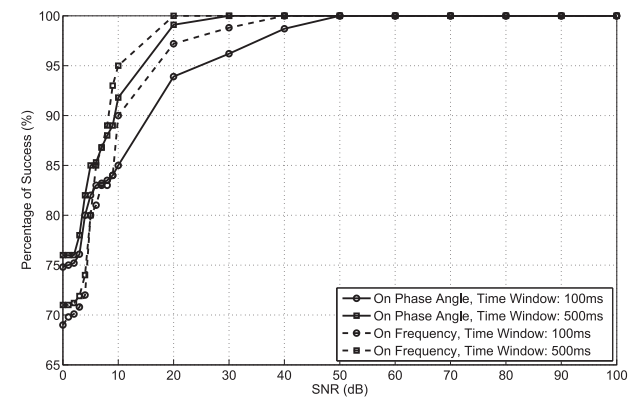

(a)

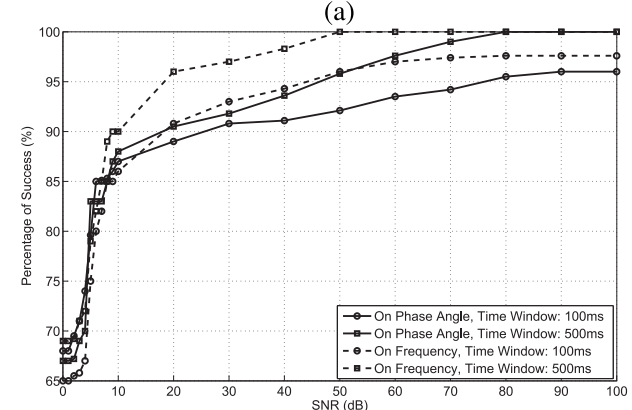

(b)

Fig. 8. Percentage of success versus SNR value with (a) fault on Line 21-22 and (b) fault on Line $14-15$.

$500 \mathrm{~ms}$, however, this method achieves interarea oscillations $0.25 \mathrm{~Hz}$ and $0.81 \mathrm{~Hz}$ to correctly identify $\left\{G_{1}\right\},\left\{G_{2}, G_{3}\right\}$, and $\left\{G_{4}, \ldots, G_{10}\right\}$. Thus, this case also reveals a limitation when the observation window is too small.

For the method of [7] with time window of $100 \mathrm{~ms}$, we calculate the phase angle comparison to incorrectly conclude the result of $\left\{G_{1}\right\},\left\{G_{2}, G_{3}, G_{4}, G_{5}, G_{6}, G_{7}\right\}$, and $\left\{G_{9}, G_{10}\right\}$. For a time window of $500 \mathrm{~ms}$, we obtain $\left\{G_{1}\right\},\left\{G_{2}, G_{3}\right\}$, $\left\{G_{4}, \ldots, G_{8}, G_{10}\right\}$, and the grouping for $G_{9}$ is ambiguous, since it can be either grouped into the second or third group. For the technique of [9], we plot the distribution of amplitude coefficients and initial phases for the dominant KMs and identify the generator coherency incorrectly as $\left\{G_{1}\right\},\left\{G_{2}, \ldots, G_{10}\right\}$ for a $100 \mathrm{~ms}$ time window. For a time window of $500 \mathrm{~ms}$, we correctly determine the coherency as $\left\{G_{1}\right\},\left\{G_{2}, G_{3}\right\}$, and $\left\{G_{4}, \ldots, G_{10}\right\}$.

These results illustrate that in order to ensure accuracy, the modal analysis-, STFT-, and KM-based methods all require sufficiently long observation windows, which may be impractical for emerging smart grid systems that exhibit extremely short time scales before reaction is required.

\section{B. White Noise}

To account for nonidealities, we evaluate the robustness of our proposed generator coherency identification technique to environmental white noise. We consider the same faults on Line $21-22$ and Line $14-15$ as in Section IV-A and an observation window of $100 \mathrm{~ms}$. In each case, we evaluate our performance for various signal-to-noise ratios (SNRs) by running 100 independent trials for each value of SNR. Moreover, we consider the situation that there is white noise on the generators' phase angle and frequency measurements.

1) Fault on Line 21-22: Fig. 8(a) shows the success rate versus SNR value from 1 to $100 \mathrm{~dB}$. From Fig. 8(a), 


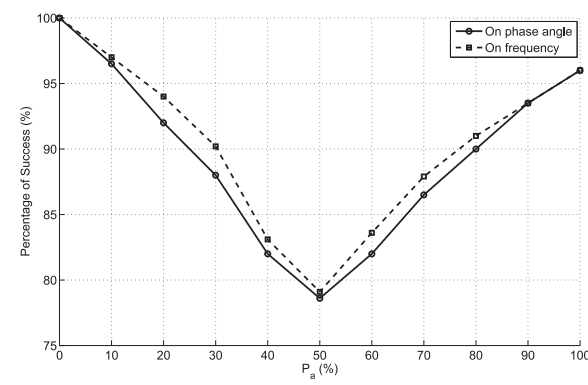

(a)

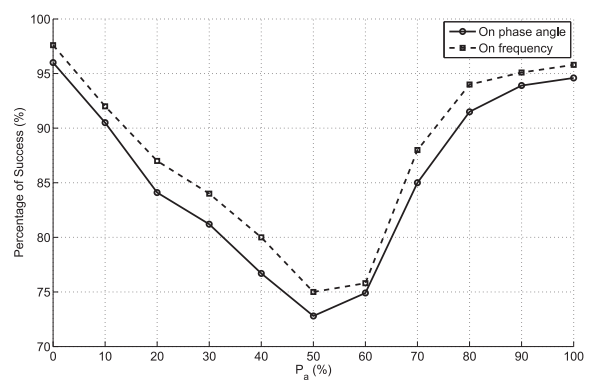

(b)

Fig. 9. Percentage of success versus percentage of PMUs being attacked $P_{a}$ with (a) fault on Line $21-22$ and (b) fault on Line $14-15$.

we observe the trade-off between high robustness at the high noise level and the high accuracy at the low noise level in our technique by considering the historical feature and dynamics of the traveling boids. When the noise level is relatively low (i.e., $S N R \geq 10 \mathrm{~dB}$ ), our proposed generator coherency identification technique is more robust to white noise on the measurement of frequency than that of phase angle. When the noise on the measurement of phase angle or frequency has $S N R \geq 10 \mathrm{~dB}$, our proposed technique achieves the percentage of success above $85 \%$. Even when the noise level is very high (i.e., $S N R=1 \mathrm{~dB}$ ), our proposed technique is still able to guarantee the percentage of success above $69 \%$.

2) Fault on Line 14-15: The success rate versus SNR value from 1 to 100 are, shown in Fig. 8(b). Here, we observe that by considering the historical feature and dynamics of the traveling boids, our proposed technique has the trade-off between high robustness (for low SNR) and the high accuracy (for high SNR). Our technique achieves the percentage of success above $85 \%$ for $S N R \geq 10 \mathrm{~dB}$. For high noise level $S N R=1 \mathrm{~dB}$, our technique is able to guarantee the percentage of success above $65 \%$. Furthermore, Fig. 8(b) also shows that when $S N R \geq 20 \mathrm{~dB}$ our proposed technique is more robust to the noise on the frequency measurement than that on the phase angle measurement.

\section{Measurement Environment With False Data Injection}

We evaluate the robustness of our technique to the popular false data injection attack on PMU measurements defined as follows.

1) Attack Model: The opponent is able to corrupt $P_{a}$ percentage of the generator bus PMUs by biasing the phase angle readings with error $e_{1}=0.5 \mathrm{rad}$ and frequency readings with error $e_{2}=1$. We consider the same fault test cases as Section IV-A and set the observation time window length as $100 \mathrm{~ms}$.

a) Fault on Line 21-22: Fig. 9(a) shows the success rate versus percentage of PMU being attacked, $P_{a}$, on phase angle and frequency, respectively. From Fig. 9(a), we observe that the success rate is worst when the attacker attempts to attack 50\% PMU readings under the attack model we considered. This is expected because by assuming the same bias on the attacked PMU readings, the relative features between biased readings are maintained. If most of the readings are biased, then their interactions will be the same as when they

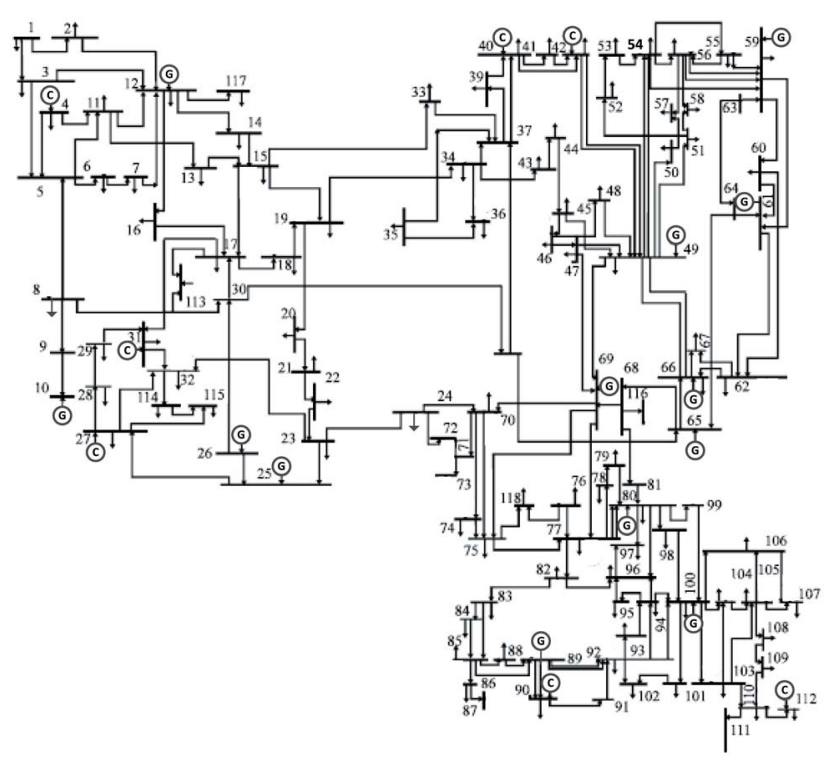

Fig. 10. Modified IEEE 118-bus test system.

TABLE VI

Generator Dynamic Parameters AND Initial Conditions For the Modified IEEE 118-Bus TEST System

\begin{tabular}{c|c|c|c|c|c|c|c|c|c|c}
\hline Unit No. & 1 & 2 & 3 & 4 & 5 & 6 & 7 & 8 & 9 & 10 \\
\hline Bus No. & 4 & 10 & 12 & 25 & 26 & 27 & 31 & 40 & 42 & 49 \\
\hline \hline Unit No. & 11 & 12 & 13 & 14 & 15 & 16 & 17 & 18 & 19 & 20 \\
\hline Bus No. & 59 & 61 & 65 & 66 & 69 & 80 & 89 & 90 & 100 & 112 \\
\hline
\end{tabular}

are not biased. Fig. 9(a) also indicates that, in the worst case under the considered attack model, our propose method guarantees the percentage of success above $78 \%$ when the PMU phase angle readings are corrupted and guarantees $79 \%$ success when the PMU frequency readings are corrupted.

b) Fault on Line 14-15: Fig. 9(b) shows the success rate versus percentage of PMUs being attacked on phase angle and frequency, respectively. From Fig. 9(b), we observe that under the worst situation when 50\% PMU readings are attacked, our proposed method guarantees the percentage of success above $72 \%$ when the phase angle PMU readings are attacked and guarantees success above $75 \%$ when the frequency PMU readings are corrupted.

Thus the above studies illustrate that our proposed measurement-based technique is robust to false data injection. Such resilience stems from our use of an information 


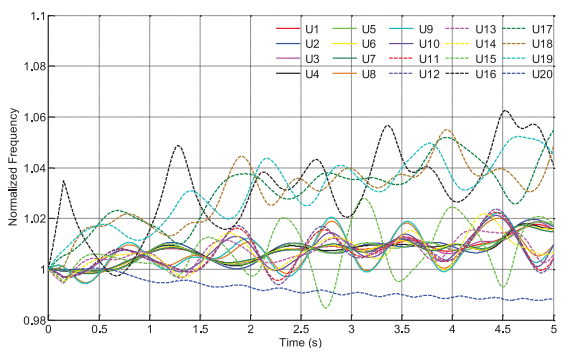

(a)

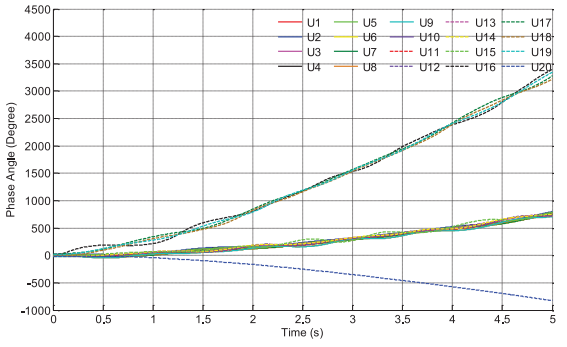

(b)

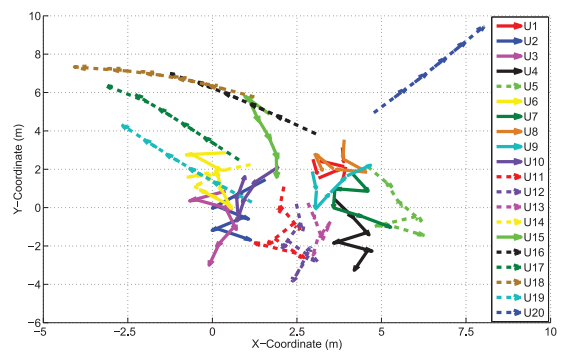

(c)

Fig. 11. (a) Normalized rotor frequencies versus time. (b) Phase angles versus time. (c) Trajectories of the boids in the information space.

space that models generator dynamics via flocking interactions. Such interactions are tolerant to false data injection, which constitutes an interruption of the corrupted dynamics. Thus our approach is able to mitigate the impact of false data injection more successfully.

\section{Larger Test System}

We evaluate the performance of our generator coherency technique on a modified IEEE 118-bus test system shown in Fig. 10; such a system represents a more complex larger scale model upon which to study performance. We employ PMUs with a sampling frequency of $50 \mathrm{~Hz}$. As suggested in [25] and [30]-[32], the standard IEEE 118-Bus test system, which is designed for reliability studies [33], is modified for transient stability studies by replacing the generator and synchronous condensers of less than 50 MVA rating with appropriate constant impedances. As shown in Fig. 10, there are 20 synchronous generating units left after the modification. The units' associated buses are listed in Table VI.

We assume that there is a three-phase short circuit fault occurring in the middle of Line $68-81$ at $t=0$. Line $68-81$ is opened at $t=150 \mathrm{~ms}$. The generators' normalized rotor frequencies and phase angles over a period of $5 \mathrm{~s}$ are shown in Fig. 11(a) and (b), respectively. We observe the following groupings: $\left\{U_{1}, \ldots, U_{15}\right\},\left\{U_{16}, \ldots, U_{19}\right\}$, and $\left\{U_{20}\right\}$.

Since transient stability maintenance is time-critical, there is only a brief observation period to determine the generator coherency. Fig. 11(c) presents the boid trajectories over one trial for an observation window length $T=100 \mathrm{~ms}$ starting immediately after the fault is cleared and using the parameters of Table IV; the dominant group result is achieved for this trial. The arrows on each boid trajectory indicates the velocity direction of each boid. From the trajectories shown in Fig. 11(c), we observe that the boids associated with the synchronous units $\left\{U_{1}, \ldots, U_{15}\right\}$ travel together as well as those associated with $\left\{U_{16}, \ldots, U_{19}\right\}$. Thus, we visually conclude that the boids constitute three flocks identifying coherent synchronous unit groups. Fig. 11 demonstrates that our proposed coherency identification technique achieves high performance for the modified IEEE 118-Bus test system, which is much more complicated compared to the New England 39-Bus test system.

To demonstrate performance when only select generators have PMUs, Fig. 12 shows the trajectories for one trial achieving the dominant boid grouping result. It is assumed that

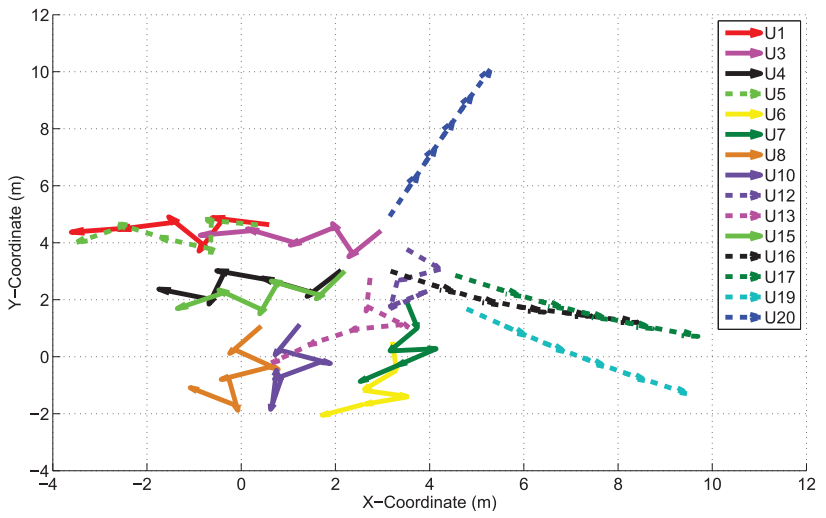

Fig. 12. Trajectories of the boids in the information space when 5 randomly selected PMUs are not available.

PMUs for $U_{2}, U_{9}, U_{11}, U_{14}$, and $U_{18}$ are unavailable. It is clear from the measurement-based nature of our approach that we cannot group generators without state estimates. However, the interactions amongst synchronous generators are mutually independent hence absent PMU readings do not impact coherency identifications for those generators with PMU readings available.

\section{CONCLUSION}

We propose a novel measurement-based multiflock technique for real-time generator coherency identification that works rapidly and accurately in the presence of serious system fault or attack. With New England 39-Bus test system, we compare the performance of our approach with existing techniques and evaluate its effectiveness in three situations: an ideal environment, a noisy environment, and an environment under cyber attack. We also evaluate our technique on the larger modified IEEE 118-Bus test system. The simulation results demonstrate how our approach achieves high identification accuracy even with very short observation windows and in the presence of environmental noise and cyber attack. Moreover, our technique does not assume specific dynamic structures for the generators enabling its adaptation and use for systems with unconventional generation sources including different fuel type synchronous machines and wind turbine synchronous generators. We believe these advantages demonstrate the potential our technique as an effective tool for rapid and robust generator coherency identification in emerging smart grid systems, especially for applications requiring maintaining transient stability of wide-area power systems. 


\section{REFERENCES}

[1] J. Hughes, "The integrated energy and communication systems architecture (IECSA) project," EPRI and Electricity Innovation Institute, Palo Alto, CA, USA, 2003.

[2] M. Klein, G. Rogers, and P. Kundur, "A fundamental study of inter-area oscillations in power systems," IEEE Trans. Power Syst., vol. 6, no. 3, pp. 914-921, Aug. 1991.

[3] R. Podmore, "Identification of coherent generators for dynamic equivalents," IEEE Trans. Power App. Syst., vol. PAS-97, no. 4, pp. 1344-1354, Jul. 1978.

[4] G. N. Ramaswamy et al., "Synchrony, aggregation, and multi-area eigenanalysis," IEEE Trans. Power Syst., vol. 10, no. 4, pp. 1986-1993, Nov. 1995.

[5] S. Yusof, G. Rogers, and R. Alden, "Slow coherency based network partitioning including load buses," IEEE Trans. Power Syst., vol. 8, no. 3, pp. 1375-1382, Aug. 1993.

[6] J. Chow, "Slow coherency and aggregation," in Power System Coherency and Model Reduction (Power Electronics and Power Systems). New York, NY, USA: Springer, 2013, pp. 39-72.

[7] M. Jonsson, M. Begovic, and J. Daalder, "A new method suitable for real-time generator coherency determination," IEEE Trans. Power Syst., vol. 19, no. 3, pp. 1473-1482, Aug. 2004.

[8] X. Wang, V. Vittal, and G. T. Heydt, "Tracing generator coherency indices using the continuation method: A novel approach," IEEE Trans. Power Syst., vol. 20, no. 3, pp. 1510-1518, Aug. 2005.

[9] Y. Susuki and I. Mezić, "Nonlinear Koopman modes and coherency identification of coupled swing dynamics," IEEE Trans. Power Syst., vol. 26, no. 9, pp. 1894-1904, Nov. 2011.

[10] N. Senroy, "Generator coherency using the Hilbert-Huang transform," IEEE Trans. Power Syst., vol. 23, no. 4, pp. 1701-1708, Nov. 2008.

[11] Phasor Measurement Unit Application Manual-RES670 ANSI 1.2, Substation Automation Products, ABB, Västerås, Sweden, Feb. 2013.

[12] B. Singh, N. Sharma, A. Tiwari, K. Verma, and S. Singh, "Applications of phasor measurement units (PMUS) in electric power system networks incorporated with facts controllers," Int. J. Eng. Sci. Technol., vol. 3, no. 3, pp. 64-82, 2011.

[13] Z. Dong et al., Emerging Techniques in Power System Analysis. Berlin, Germany: Springer-Verlag, 2010.

[14] (2013, Jun. 4). MRC Approvals 5/30/13: PMU Costs, CFTC Order, UTC Credit [Online]. Available: http://www.rtoinsider.com/mrc-approvals53013-pmu-costs-cftc-order-utc-credit/

[15] M. Glavic and T. V. Cutsem, "Investigating state reconstruction from scarce synchronized phasor measurements," in Proc. IEEE PES Trondheim PowerTech, Trondheim, Norway, Jun. 2011, pp. 1-8.

[16] E. Ghahremani and I. Kamwa, "Online state estimation of a synchronous generator using unscented Kalman filter from phasor measurements units," IEEE Trans. Energy Convers., vol. 26, no. 4, pp. 1099-1108, Dec. 2011.

[17] J. Warichet, T. Sezi, and J. C. Maun, "Considerations about synchrophasors measurement in dynamic system conditions," Int. J. Elect. Power Energy Syst., vol. 31, no. 9, pp. 452-464, Oct. 2009.

[18] P. Zhang, J. Chen, and M. Shao, "Considerations about synchrophasors measurement in dynamic system conditions," Dept. Transmiss. Distrib., Elect. Power Res. Inst., Marco Island, FL, USA, Tech. Rep. 1015511, Oct. 2007.

[19] J. Wei and D. Kundur, "A multi-flock approach to rapid dynamic generator coherency identification," in Proc. IEEE Power Energy Soc. Gen. Meeting, Vancouver, BC, Canada, Jul. 2013, pp. 1-5.

[20] C. Reynolds, "Flocks, herds, and schools: A distributed behavioral model," Comput. Graph., vol. 21, no. 4, pp. 25-34, Jul. 1987.

[21] I. Couzin, J. Krause, R. James, G. Ruxton, and N. Franks, "Collective memory and spatial sorting in animal groups," J. Theor. Biol., vol. 218, no. 1, pp. 1-11, 2002.

[22] Z. Huang and J. Dagle, "SynchroPhasor measurements: System architecture and performance evaluation in supporting wide-area applications," in Proc. 21st IEEE Power Energy Soc. Gen. Meeting Convers. Delivery Elect. Energy, Pittsburgh, PA, USA, Jul. 2008.

[23] H. Khalil, Nonlinear Systems. Upper Saddle River, NJ, USA: Prentice Hall, 2001.

[24] R. Olfati-Saber, "Flocking for multi-agent dynamic systems: Algorithms and theory," IEEE Trans. Autom. Control, vol. 51, no. 3, pp. 401-420, Mar. 2006.

[25] T. Athay, R. Podmore, and S. Virmani, "A practical method for the direct analysis of transient stability," IEEE Trans. Power App. Syst., vol. PAS-98, no. 2, pp. 573-587, Mar. 1979.

[26] I. Dobson, "Voltages across an area of a network," IEEE Trans. Power Syst., vol. 27, no. 2, pp. 993-1002, May 2012.
[27] M. Zima and D. Ernst, "On multi-area control in electric power systems," in Proc. 15th Power Syst. Comput. Conf. (PSCC), Liège, Belgium, Aug. 2005, pp. 1-8.

[28] X. Wang, "Slow coherency grouping based islanding using minimal cutsets and generator coherency index tracing using the continuation method," Ph.D. dissertation, Dept. Elect. Comput. Eng., Iowa State University, Ames, IA, USA, 2005.

[29] G. Pyo, J. Park, and S. Moon, "Coherency identification of generators using a PAM algorithm for dynamic reduction of power systems," Energies, vol. 5, no. 11, pp. 4417-4429, 2012.

[30] A. Chang and M. Adibi, "Power system dynamic equivalents," IEEE Trans. Power App. Syst., vol. PAS-89, no. 8, pp. 1737-1744, Nov. 1970.

[31] T. Athay, R. Podmore, and S. Virmani, "Transient energy stability analysis," in Proc. Eng. Found. Conf. Syst. Eng. Power, Henniker, NH, USA, Aug. 1977, pp. 21-26.

[32] Y. Wei, Z. Zheng, Y. Sun, Z. Wei, and G. Sun, "Voltage stability bifurcation analysis for AC/DC systems with VSC-HVDC," Abstr. Appl. Anal., vol. 2013, 2013, Art. ID 387167.

[33] Power System Test Case Archive [Online]. Available: http://www.ee.washington.edu/research/pstca/pf118/pg_tca118bus.htm

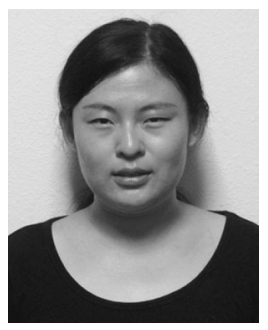

Jin Wei (S'09) received the B.E. degree in electronic information engineering from the Beijing University of Aeronautics and Astronautics, Beijing, China, in 2004; the M.S. degree in electrical engineering from the University of Hawaii at Manoa, Honolulu, HI, USA, in 2008; and the Ph.D. degree in electrical and computer engineering from the University of Toronto, Toronto, ON, Canada, in 2014.

She is an Assistant Professor in Electrical and Computer Engineering with the University of Akron, Akron, OH, USA. She was a Post-Doctoral Fellow with the National Renewable Energy Laboratory (NREL), Golden, CO, USA, in 2014. Her current research interests include the cyber-physical energy system, cyber-physical systems security, renewable energy integration, stochastic modeling and optimization of large-scale systems, and cognitive wired/wireless communication networks.

Dr. Wei has been the recipient of travel grants and is an IEEE Canadian Conference on Electrical and Computer Engineering'12 Best Student Paper Finalist.

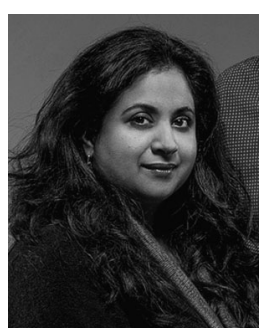

Deepa Kundur (S'03) received the B.A.Sc., M.A.Sc., and Ph.D. degrees in electrical and computer engineering from the University of Toronto, Toronto, ON, Canada, in 1993, 1995, and 1999, respectively.

She is a Professor of Electrical and Computer Engineering at the University of Toronto. Her current research interests include cyber security of the electric smart grid, cyber-physical system theory, security and privacy of sensor networks and multimedia security and forensics.

Dr. Kundur has served on the North American Reliability Corporation Smart Grid Task Force, has been on several editorial boards, and currently serves as Associate Editor of the IEEE TRANSACTIONS ON INFORMATION FORENSICS AND SECURITY. Her research has received best paper recognitions at the 2008 IEEE International Conference on Computer Communications Workshop on Mission Critical Networks, the 2011 Cyber Security and Information Intelligence Research Workshop, the 2012 IEEE Canadian Conference on Electrical and Computer Engineering, and the 2013 IEEE Power and Energy Society General Meeting.

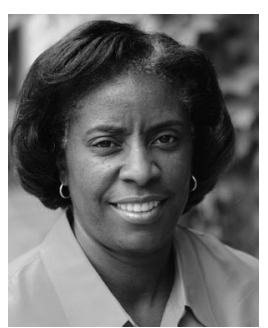

Karen L. Butler-Purry (SM'01) received the B.S. degree (summa cum laude) in electrical engineering from Southern University, Baton Rouge, LA, USA, in 1985; the M.S. degree in electrical engineering from the University of Texas at Austin, Austin, TX, USA, in 1987; and the Ph.D. degree in electrical engineering from Howard University, Washington, DC, USA, in 1994.

She joined Texas A\&M University, College Station, TX, in 1994, where she currently serves as Associate Provost for Graduate Studies and as a Professor with the Department of Electrical and Computer Engineering. Her current research interests include distribution automation, cyber physical systems protection, and intelligent systems for power quality, equipment deterioration, and fault diagnosis. 